\title{
The 39th international conference of the EURO working group on operational research applied to health services: ORAHS 2013 special issue
}

\author{
Tuğba Çayırlı ${ }^{1}$ - Murat M. Günal ${ }^{2} \cdot$ Evrim Güneș $^{3} \cdot$ Lerzan Örmeci $^{3}$
}

Received: 11 March 2015 / Accepted: 18 March 2015 / Published online: 31 March 2015

(C) Springer Science+Business Media New York 2015

\section{ORAHS 2013}

The healthcare sector is facing major challenges worldwide in terms of higher demands for efficiency, quality and equity. Operational Research (OR) techniques offer valuable tools for improving the design and delivery of healthcare services (e.g., inpatient and outpatient care, admission processes, emergency services, home healthcare, etc.), as well as improving the decision-making processes and implementation of public health policies.

The EURO Working Group on Operational Research Applied to Health Services (ORAHS) was formed in 1975 as a special interest group within the European branch (EURO) of the International Federation of Operational Research Societies (IFORS) [1]. The group holds international conferences every year. The goal is to bring together the academicians and practitioners for disseminating ideas, knowledge and research on the application of OR methods to healthcare.

Murat M. Günal

mgunal@dho.edu.tr

Tuğba Çayırlı

tugba.cayirli@ozyegin.edu.tr

Evrim Güneș

egunes@ku.edu.tr

Lerzan Örmeci

lormeci@ku.edu.tr

1 Özyeğin University, İstanbul, Turkey

2 Turkish Naval Academy, İstanbul, Turkey

3 Koç University, İstanbul, Turkey
The 39th ORAHS conference was held at Koç University in Istanbul, Turkey during July 7-12 in 2013. The organizing committee included Tuğba Çayırlı from Özyeğin University, Murat Günal from Turkish Naval Academy, Evrim Güneş and Lerzan Örmeci from Koç University, and Yasar A. Özcan from Virginia Commonwealth University. The conference theme was "OR for New Challenges in Healthcare Services". There was one keynote talk, two panel discussions and three tutorial sessions, in addition to the regular oral and poster presentations. These events included the following details (in order of presentation):

- Panel Discussion 1: "Healthcare Reform and New Challenges in Turkish Healthcare System and Operational Research" Speakers: Prof. Dr. Șevket Ruacan (Medical School at Koç University), Prof. Hacer Özgen Narcı (Istanbul Medipol University), and Prof. Dr. Sabahattin Aydın (Istanbul Medipol University).

- Tutorial Session 1: "Healthcare Logistics" by Prof. Stefan Nickel (Karlsruhe Institute of Technology Institute of Operational Research).

- Keynote Talk: "New and Old Forces are Shaping Major Changes in Health Care Delivery Presenting Great Opportunities for OR in Health Care" by Prof. William P. Pierskalla (The Anderson School at UCLA - The Wharton School, University of Pennsylvania).

- Tutorial Session 2: "Health Policy Modeling" by Prof. Margaret Brandeau (Stanford University).

- Panel Discussion 2: "Future of Healthcare: Challenges for Operational Research" Speakers: Prof. William P. Pierskalla (The Anderson School at UCLA - The Wharton School, University of Pennsylvania), Prof. Yasar A. Ozcan (Department of Health Administration, Virginia Commonwealth University), Prof. Marion Rauner (School of 
Business, Economics and Statistics, University of Vienna), Prof. Mike Pidd (The Management School, Lancaster University), and Tom Bowen (The Balance of Care Group, U.K.).

- Tutorial Session 3: "Stochastic Disease Modeling and Chronic Disease Management" by Prof. Mariel Lavieri (University of Michigan).

ORAHS'2013 conference hosted 139 participants from 28 different countries across the world with 96 papers presented in total. It was sponsored by EURO, Koç University, Özyeğin University and Mado-Turkey.

\section{HCMS special issue}

This special issue of Health Care Management Science is devoted to papers presented at ORAHS 2013 conference. In total, 25 papers were submitted for consideration at this special issue and after a careful review process 12 papers were accepted for publication. The papers in this issue can be grouped into two broad categories, each including six papers. The first category is on the general area of hospital operations, while the second category focuses on preventive health care models and decision making.

\subsection{Hospital operations}

Khaniyev et al. provide a statistical model to adjust an existing surgery duration estimation method by accounting for additional temporal, operational, and staff related factors. This method improves the accuracy of the estimates by eliminating bias and reducing large errors. They also show that such adjustments can be used to improve other good prediction models in the literature.

Mallor et al. propose three types of discharge rules for an intensive care unit (ICU) with the aim of producing a set of, so-called, target service rates that depend on the number of patients in the ICU. The target service rates are determined by a theoretical model that combines queueing theory and optimization techniques in order to minimize the rate of patient rejection due to a full ICU. A simulation model is built to compare the service rates provided by these rules and the theoretical solutions. As a result, the authors are able to suggest two flexible and medically-meaningful discharge rules that achieve the target service levels.

Marques et al. investigate an elective surgery scheduling problem in a public hospital in Lisbon, Portugal. The objectives of the bi-criteria optimization problem are to minimize the waiting lists for surgery and to maximize the occupation of surgical suites. The proposed heuristic approach is shown to build good schedules illustrated with empirical data from the hospital they studied.
Meng et al. study is motivated by interventions to reduce waiting times that are closely monitored by the Ministry of Health in Singapore. More specifically, the authors study emergency departments, where patients who wait longer than a maximum wait time or threshold are processed within the target time period. They propose an efficient analytical approach for finding the new mean and variance of waiting times given the threshold period and their interaction, assuming that the waiting times follow a phase-type distribution. The goal is to facilitate policy-makers' decision-making process through testing different scenarios prior to actually implementing such interventions.

van der Veen et al. consider workforce capacity allocation. They introduce the annualized hours regime to create more flexibility in contract types and cost savings. The implementation of this idea through a mathematical formulation brings a potential decrease of $5.2 \%$ in annual personnel wages.

$\mathrm{Xie}$ et al. address personnel-scheduling in emergency department where the goal is to minimize patients' waiting times. The authors model the problem as a stochastic mixedinteger programming and solve for the optimal schedules using the Sample Average Approximation (SAA) method. The solutions are later tested for validity and robustness using discrete-event simulation based on real data collected from a hospital in France.

\subsection{Preventive healthcare models and decision making}

Davari et al. consider preventive healthcare programs, which aim to achieve a high level of public participation (which is a function of the attractiveness of a health care facility) and to provide equity among various groups. The participation is represented via a fuzzy model to represent the vagueness in the concept of attractiveness. The paper develops two fuzzy bi-objective models with a budget constraint, and proposes two solution methodologies. The results are illustrated through a case study in Istanbul, Turkey.

Karimi et al. present a health behavior model to explore alternative protective measures to vitiate influenza outbreaks. They work on a university campus with students who either caught influenza virus or have proximity. Authors use Agent Based Simulation (ABS) methodology but implement it in a well-known Discrete Event Simulation (DES) software. The source of data for modelling human behavior is a questionnaire targeting to investigate knowledge level of people about influenza. Use of questionnaire in ABS models is spreading out quite fast in the literature and apparently it is depicting human behavior quite well.

Kok et al. uses System Dynamics (SD), another wellestablished method in healthcare policy making, to optimize HIV testing programs in Canada. Their model is built 
upon causal loop diagrams and is coupled with a nonlinear compartmental disease model for the HIV epidemic. To build the SD model, a system of differential equations is written which corresponds to each compartment in HIV care continuum. Data sources in this study are local centers and published literature. After calibrating and validating model parameters, they run the model for various scenarios in which resource allocations to testing programs are altered. Results show that optimal resource allocation strategy favors routine testing in high prevalence settings. The project team closely collaborated with local authorities and apparently the results affected decision making in HIV testing strategy.

Lee et al. investigate the allocation of screening resources across a population at risk for developing Hepatocellular Carcinoma (HCC). This risk is a function of his/her biomedical dynamics that must be learned by the system over time. The authors develop three classes of reinforcement learning policies, and compare their performances with current practice via a simulation based on a data set with 1050 patients. The study demonstrates the effects of resource levels on the benefits of learning-based screening policies and provides metrics of policy performance.

In Sadat et al., two hospitals serve a single class of patients by providing the same type of service with different service rates. Moreover, their perceived quality of care is different. Patients choose one of the hospitals to maximize their own utility which is a function of price and the patients' perceived quality of life during their life expectancy. The competition between the two hospitals is analyzed in various parameters setting. The settings in which a Nash equilibrium can be identified are characterized, whereas the remaining cases are investigated numerically.

Scherrer et al. develop a formulation for a Decision Support System (DSS) which can evaluate options for breast cancer treatment. They indicate that therapy planning is a sequential multi-criteria decision making problem since there are around 310 attributes related to status of patient case at any point in time and 190 attributes related to treatment and diagnostic of non-metastasized breast cancer. Their comprehensive data model and mathematical notation allow for the automated and time-efficient search for suitable therapy options, which then formed the basis for a software tool.

\section{Conclusion}

ORAHS 2013 was a successful conference in two ways. First, the conference hosted world-renowned scholars who delivered keynote speeches and tutorials. These talks covered a broad range of interests in the field; from public health and disease modeling to hospital operations. As such, these talks generated very high interest from all the participants. Moreover, they provided an excellent overview for young researchers who are at the early stages of their career.

Second, having a high number of participants from many countries around the world created a perfect medium for international collaboration on healthcare research. The panel discussions demonstrated fruitful discussions from participants with diverse national experiences.

This issue aims at contributing to the knowledge in Operational Research/Management Science (OR/MS) in healthcare. The diversity of the topics in these papers is an indication of content richness. The first group of papers summarized under hospital operations offer valuable insights on how to improve planning and management for problems such as surgical scheduling, designing discharge rules for intensive care units, personnel-scheduling and workforce capacity allocation. Other theoretical papers focus on improving the accuracy of surgery duration estimates, as well as improving interventions for wait time reductions. The second group contributes to the understanding of preventive healthcare programs with different objectives and of preventive actions such as informing potential patients about a sickness, or developing screening policies for serious sicknesses. The other two papers consider decision making in the context of treatment and individual patient utilities.

All of these studies are highly relevant to practice and most of them also use empirical data from healthcare settings to validate their models and/or implement their solution methodologies. A wide range of methodologies are included in both groups, such as simulation, system dynamics, queuing theory, optimization, and analytical/statistical modelling.

Overall, we can conclude that the growing field of healthcare OR/MS offers valuable tools and approaches for improving decision-making in healthcare. This special issue includes a set of papers that we believe is a good representative of research based on real problems that are rigorously studied. It covers a wide range of universal problems that are illustrated with healthcare settings in Portugal, France, Singapore and Turkey, among others. As such, these papers well illustrate how academic research can strengthen the link between theory and practice for improving the quality of care worldwide.

\section{Reference}

1. ORAHS (2015) http://orahs.di.unito.it/about.html (As accessed on March 2015. 